\title{
O PAPEL DA CIRURGIA TORÁCICA VÍDEO-ASSISTIDA-CTVA- NO TRATAMENTO DA PNEUMONITE NECROSANTE NA CRIANÇA
}

\author{
THE ROLE OF VIDEO-ASSISTED THORACOSCOPIC SURGERY - VATS - IN THE \\ MANAGEMENT OF NECROTIZING PNEUMONITIS IN CHILDREN
}

Carlos Eduardo Prieto Velhote, TCBC-SP1; Manoel Carlos Prieto Velhote, ACBC-SP²

\begin{abstract}
RESUMO: Objetivo: Avaliar o papel da cirurgia torácica vídeo-assistida (CTVA) no tratamento das complicações pleurais da pneumonite necrosante $(\mathrm{PN})$ na criança, em sua fase aguda. Método: Avaliação prospectiva de sete pacientes com idades entre quatro e sete anos, portadores de empiema pleural com PN, com diagnóstico confirmado por tomografia computadorizada de tórax (TC) e cirurgia. Realizou-se a descorticação pulmonar com esvaziamento do pleuris, desbridamento e ressecção do tecido pulmonar comprometido, e drenagem torácica, através de videotoracoscopia com três portais. Foram avaliados no pós-operatório a curva térmica, o leucograma, o tempo de drenagem torácica e a permanência hospitalar. Resultados: Houve re-expansão pulmonar completa em todos os pacientes tratados pela CTVA, com rápida melhora do estado geral, queda de temperatura e da leucocitose na primeira semana pós-tratamento. O período de drenagem pós-CTVA variou de sete a 17 dias, e a permanência hospitalar póscirúrgica foi menor quando comparada com dados da literatura em que o tratamento utilizado foi a drenagem torácica com antibioticoterapia ou a cirurgia a céu aberto. Conclusões: A utilização da CTVA como coadjuvante no tratamento da PN acompanhada de complicações pleurais, tem papel importante na aceleração da recuperação clínica do paciente, proporcionando a reexpansão mais rápida do pulmão colapsado, com melhoria das condições respiratórias (Rev. Col. Bras. Cir. 2006; 33(1): 11-14).
\end{abstract}

Descritores: Pneumonia; Necrose; Empiema Pleural; Cirurgia Torácica Vídeo-assistida; Criança.

\section{INTRODUÇÃO}

De tratamento fundamentalmente clínico, a pneumonite necrosante (PN) é uma complicação pouco freqüente da pneumonia lobar ${ }^{1}$. Caracteriza-se pela necrose e liquefação do tecido pulmonar com formação de múltiplas cavidades não coalescentes ${ }^{1,2}$, e que, acompanhada de complicações pleurais, exige a adoção de medidas mais agressivas para abreviar seu tratamento $0^{3-6}$. Uma vez que a radiografia simples de tórax é insuficiente para a perfeita avaliação das condições pulmonares e pleurais destes pacientes, os portadores de empiema pleural com colapso pulmonar ou fístulas bronco-pleurais de evolução arrastada, devem ser avaliados pela TC para a adequação do tratamento ${ }^{5,7}$.

Como método terapêutico coadjuvante, a minitoracotomia com desbridamento e ressecção cirúrgica do tecido necrótico, sutura das áreas cruentas e drenagem da cavidade torácica, tem sido aceita, com restrições, quer por ser considerada um procedimento muito agressivo, quer pelo desconforto causado pela dor pós-operatória ${ }^{8,9}$. Com o advento de procedimentos minimamente invasivos, a cirurgia torácica vídeo-assistida (CTVA) tornou-se, em muitos Serviços, o procedimento diagnóstico e terapêutico de escolha nos casos suspeitos de PN ${ }^{3,5,9,10}$.

Expomos aqui nossa experiência inicial na utilização da CTVA no tratamento de crianças portadoras de PN.

\section{MÉTODO}

O estudo considerou pacientes portadores de pneumonia grave, submetidos a diferentes esquemas terapêuticos, e que evoluíram com piora clínica e formação de derrame pleural. Foram avaliados de forma prospectiva sete pacientes, sendo três do sexo masculino e quatro do sexo feminino, com idades variando entre quatro e sete anos, admitidos com quadro pneumônico associado ao empiema pleural (seis pacientes) ou pio-pneumotórax (um paciente). O comprometimento pulmonar e pleural ocorreu no hemitórax esquerdo em três pacientes, no hemitórax direito em três pacientes e foi bilateral em um paciente.

Confirmado o diagnóstico de empiema pleural pela punção torácica, procedeu-se à drenagem do tórax com dreno tubular, e à antibioticoterapia com a associação de vancomicina à ceftrioxona. Nos pacientes com evolução desfavorável (febre alta, toxemia e leucocitose acentuada) realizou-se TC, por volta do $10^{\circ}$ dia pós-drenagem, para avaliação do quadro pulmonar e pleural (Figura 1). Caracterizada PN com complicações pleurais, os pacientes foram submetidos à CTVA sob anestesia geral com enflurane e entubação seletiva, colocando-se o paciente em decúbito lateral com o lado acometido para cima, sem coxim sob o gradeado costal. Nos pacientes em que não foi possível a entubação seletiva, manteve-se pressão intrapleural positiva com $\mathrm{CO}_{2}$ no hemitórax afetado,

1. Especialista em Cirurgia Pediátrica pela Sociedade Brasileira de Cirurgia Pediátrica; Cirurgião Pediátrico do Hospital Carlos Chagas.

2. Assistente Doutor da Disciplina de Cirurgia Pediátrica do Instituto da Criança do Hospital das Clínicas da Universidade de São Paulo; Cirurgião Pediátrico do Hospital Carlos Chagas. 
através de um insuflador convencional em baixa pressão (4 a 6 $\mathrm{cmH}_{2} \mathrm{O}$ ). Foi utilizada ótica de $10 \mathrm{~mm} / 30^{\circ}$, introduzida diretamente, ou através de um trocarte, pelo orifício original de drenagem. Após o descolamento parcial da pleura visceral por dissecção romba, foram introduzidos mais dois trocartes de 5 $\mathrm{mm}$ ou $10 \mathrm{~mm}$, em pontos previamente escolhidos (Figura 2), para as pinças de trabalho ${ }^{10,11}$. Procedeu-se ao desbridamento e à ressecção do parênquima pulmonar necrótico e ao esvaziamento do pleuris fibrinoso, com drenagem do espaço pleural com dreno tubular, único e multifenestrado, em selo d'água, colocado sob visão direta. Para a ressecção do tecido pulmonar desvitalizado foram utilizados o bisturi elétrico com "hook" ou o Ultracision ${ }^{\circledR}$ de $5 \mathrm{~mm}$, com ampla exposição das áreas escavadas para a cavidade pleural.

\section{RESULTADOS}

A duração média do procedimento foi de 80 minutos (Mínimo: 60; Máximo: 120). Todos os pacientes estudados tiveram seus procedimentos realizados exclusivamente por CTVAe não houve nenhuma conversão para procedimento a céu aberto. Já no pós-operatório imediato houve melhora no estado geral e na dor torácica dos pacientes, com queda progressiva da temperatura e da leucocitose nas primeiras 48 horas e normalização destes parâmetros até o $4^{\circ}$ dia de pós-operatório (Tabela 1). A retirada do dreno torácico foi feita entre o $7^{\circ}$. e o $17^{\circ}$. dias de pós-operatório, quando não mais havia drenagem de secreção ou escape de ar pelo mesmo. A única exceção se deu na paciente portadora de PN bilateral, em que a drenagem fechada foi transformada em drenagem aberta no $15^{\circ}$. dia de pós-operatório, por persistência de fístula broncopleural. A drenagem aberta foi mantida por três dias, quando o dreno foi finalmente retirado, e a fístula fechada espontaneamente após quatro dias.

\section{DISCUSSÃO}

Classicamente definida como secundária a infecções pulmonares por Streptococus pneumoniae, a PN também pode estar associada a pneumonias causadas por outros agentes etiológicos como Streptococus viridans, Staphylococus aureus e Haemophilus influenzae entre outros ${ }^{3}$. Com comprometimento do estado geral e febre alta, estas crianças frequentemente apresentam hemograma do tipo infeccioso com leucocitose acentuada. A floculação e septação do empiema detectadas ao ultra-som de tórax, associada ao colapso pulmonar ou à presença de fístula bronco-pleural na radiologia simples de tórax, apesar do tratamento adequado, são sugestivas de $\mathrm{PN}^{2,3,12}$.

Os sete pacientes aqui estudados se enquadram nas premissas acima citadas. $\mathrm{O}$ exame bacteriológico da secreção pleural colhida quando do diagnóstico não foi realizado pois todos os pacientes já faziam uso de antibióticos, o que falsearia o resultado obtido ${ }^{2,8,16}$. Além disso, dados da literatura relatam percentuais de crescimento bacteriano, em cultura de aspirados pleurais, variando entre $15 \%$ a $50 \%$ após o quinto dia de incubação o que diminui o valor prático do exame $^{3,7,13}$

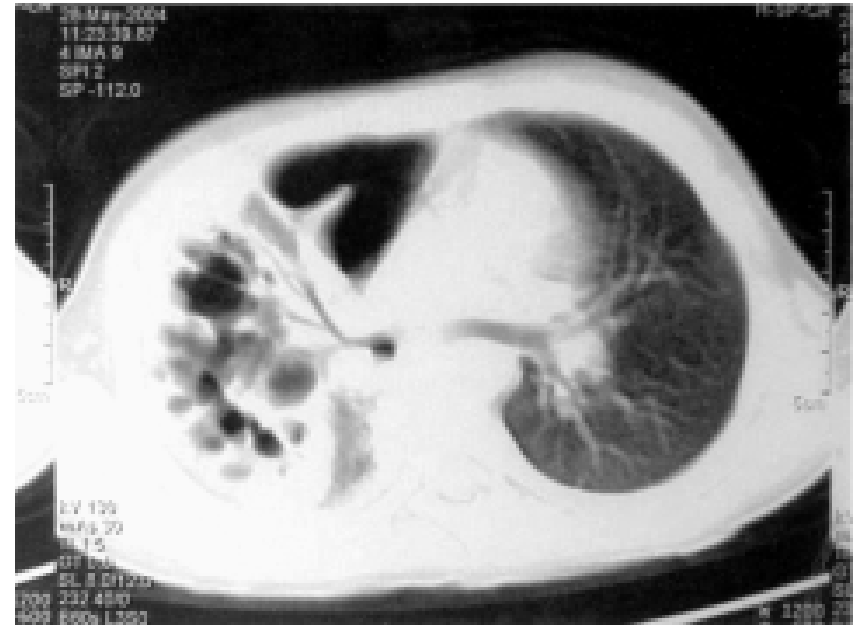

Figura 1 - Tomografia computadorizada de tórax de paciente portador de peneumonite necrosante com complicações pleurais. Observar áreas de escavação pulmonar não confluentes e pneumotórax encistado para cardíaco à direita.

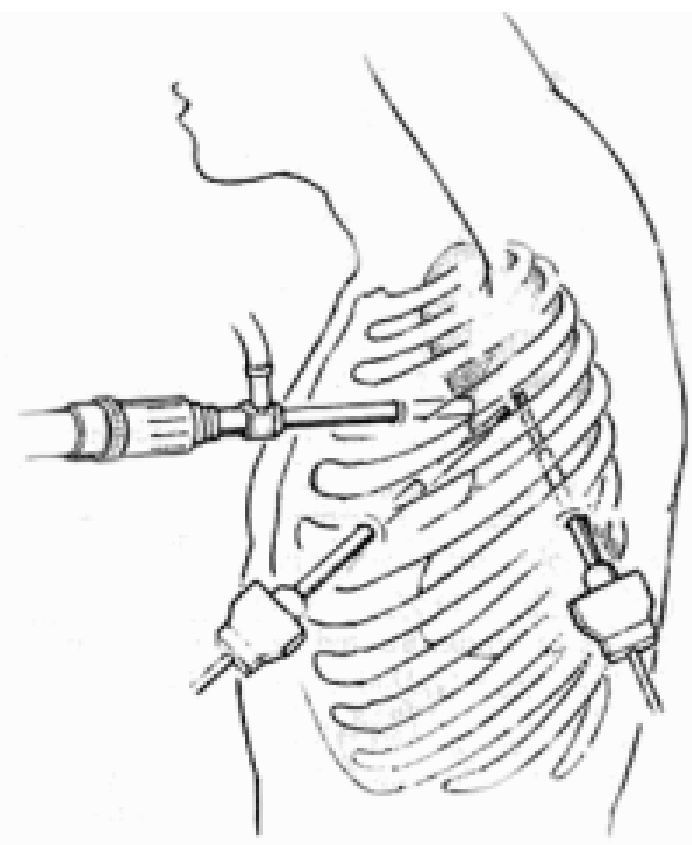

Figura 2 - Posicionamento da óptica e pinças de trabalho para o tratamento da pneumonite necrosante.

Como a organização do pleuris fibrinoso ocorre entre a $2^{\text {a. }}$ e $3^{\text {a. }}$ semanas da instalação do empiema, optou-se pela avaliação das condições pleuro-pulmonares de pacientes suspeitos de PN pela TC por volta do décimo dia pós-drenagem ${ }^{12}$. Assim, com a confirmação radiológica da PN, foi possível instituir o tratamento cirúrgico pela CTVA entre o décimo primeiro e décimo sexto dias pós-diagnóstico, tornando o desbridamento cirúrgico mais fácil e eficaz ${ }^{6,713,14}$. Em todos os pacientes foi possível a realização do desbridamento cirúrgico, sem necessidade da sua conversão para procedimento a céu aberto.

A anestesia com entubação seletiva, apesar de mais trabalhosa quando há necessidade de se entubar o brônquio fonte esquerdo, facilita sobremaneira a manipulação vídeo- 
Tabela 1 - Distribuição de pacientes portadores de pneumonite necrosante com complicações pleurais, submetidos a cirurgia torácica video-assistida (CTVA).

\begin{tabular}{cccccccccc}
\hline Paciente & $\begin{array}{c}\text { Idade } \\
\text { (anos) }\end{array}$ & Pulmão & \multicolumn{2}{c}{$\begin{array}{c}\text { Pré-CTVA } \\
\text { Temp }\left({ }^{\circ} \mathbf{C}\right) \text { Leucócitos Drenagem** }\end{array}$} & \multicolumn{2}{c}{$\begin{array}{c}\text { Pós-CTVA }\left(4^{\mathbf{0}} \mathbf{P O}\right) \\
\text { Temp }\left({ }^{\circ} \mathbf{C}\right) \text { Leucócitos Drenagem** }\end{array}$} & $\begin{array}{c}\text { Permanência } \\
\text { Hospitalar } \\
\text { Pós-CTVA* }\end{array}$ \\
\hline 1 & 5 & Esquerdo & $39,2^{\circ} \mathrm{C}$ & 23.700 & 15 & $37,6^{\circ} \mathrm{C}$ & 10.500 & 7 & 10 \\
2 & 5 & Esquerdo & $39,6^{\circ} \mathrm{C}$ & 24.800 & 12 & $37,7^{\circ} \mathrm{C}$ & 10.600 & 10 & 16 \\
3 & 4 & Direito & $38,6^{\circ} \mathrm{C}$ & 25.800 & 12 & $36,6^{\circ} \mathrm{C}$ & 10.400 & 13 & 15 \\
4 & 4 & Direito & $39,0^{\circ} \mathrm{C}$ & 22.100 & 12 & $38,0^{\circ} \mathrm{C}$ & 15.300 & 17 & 33 \\
5 & 3 & Esquerdo & $37,8^{\circ} \mathrm{C}$ & 15.600 & 16 & $36,8^{\circ} \mathrm{C}$ & 10.200 & 10 & 16 \\
6 & 4 & Direito & $38,8^{\circ} \mathrm{C}$ & 25.500 & 11 & $37,6^{\circ} \mathrm{C}$ & 12.300 & 12 & 15 \\
7 & 7 & Direito & $38,8^{\circ} \mathrm{C}$ & 22.600 & 12 & $37,4^{\circ} \mathrm{C}$ & 11.000 & 7 & 10 \\
8 & & Esquerdo & $39,8^{\circ} \mathrm{C}$ & 42.700 & 10 & $37,2^{\circ} \mathrm{C}$ & 13.100 & 8 & 10 \\
\hline
\end{tabular}

endoscópica do pulmão comprometido. Na sua impossibilidade, a injeção de $\mathrm{CO}_{2}$ em baixa pressão mantendo pressão positiva na cavidade torácica afetada, compensa parcialmente as dificuldades técnicas decorrentes da mobilidade pulmonar. A ressecção do tecido necrosado é ponto importante do tratamento, pois permite a recuperação mais rápida da área lesada pela melhora das condições locais.

O procedimento é naturalmente de longa duração, tedioso, pois o campo é exíguo, inflamado, e a criança tem lesão pulmonar grave o que diminui sua reserva pulmonar. Apesar disso, fora ocasionais quedas na saturação de oxigênio compensadas pela parada temporária do procedimento, não houve maiores dificuldades na sua realização.

A escolha do $4^{\circ}$. dia de pós-operatório para avaliação da temperatura e da leucocitose se deveu ao fato de estudos anteriores demonstrarem que tais parâmetros se normalizam entre 48 e 72 horas pós-debridamento a céu aberto ${ }^{3}$, dados estes coincidentes com os obtidos no presente estudo. As- sim, com a melhora progressiva do quadro clínico e radiológico foi possível a retirada do dreno torácico entre sete e dez dias após a cirurgia, exceto na paciente com fístula broncopleural pós-operatória (Tabela 1).

Os dados de nossos pacientes portadores de PN com complicação pleural se superpõem aos da literatura quanto à permanência, tempo de drenagem pós-operatória e resultado final, em casos de empiema pleural tratados por CTVA ${ }^{13-15}$.

Os controles radiológicos a médio e longo prazo mostram recuperação quase total das lesões, sobrando como seqüela tão somente algumas cicatrizes no parênquima pulmonar.

Apesar do pequeno número de pacientes, nossa casuística sugere que o tratamento da PN por vídeo-cirurgia é tão eficiente quanto aquele propiciado pela cirurgia a céu aberto, com vantagem de causar menos dor no pós-operatório, ter um resultado estético superior, além de propiciar uma menor permanência hospitalar.

\begin{abstract}
Background: To evaluate the role of video-assisted thoracoscopic surgery (VATS), on children with pleural complications of necrotizing pneumonitis (NP). Methods: Prospective evaluation of seven patients aged from four to seven years, with necrotizing pneumonitis and pleural empyema diagnosed by computerized thoracic scan. They were treated by debridment, resection of the compromised lung tissue and thoracic drainage, using a three port video-assisted surgery. Results: Expansion of the incarcerated lung, disappearance of fever and leucocytosis within the first postoperative week. The chest tube stayed in place from 7 to 17 days. Conclusion:

Although there are only few patients in this series, our study suggest that VATS is as efficient in the treatment of NP as open surgery. VATS also causes less pain and allows a shorter hospital stay when compared to open surgery.
\end{abstract}

Key words: Pneumonia; Necrosis; Empyema, pleural; Thoracic surgery, video-assisted; Child.

\section{REFERÊNCIAS}

1. Chen KC, Su YT, Lin WL, et al. Clinical analysis of necrotizing pneumonia in children: three-year experience in a single medical center. Acta Pediatr Taiwan. 2003;44(6):343-8.

2. Hoffer FA, Bloom DA, Colin AA, et al. Lung abscess versus necrotizing pneumonia: implications for interventional therapy. Pediatr Radiol. 1999;29(2):87-91.

3. Wong KS, Chiu CH, Yeow KM, et al. Necrotising pneumonitis in children. Eur J Pediatr. 2000;159(9):684-8.
4. McCarthy VP,Patamasucon P, Gaines T, et al. Necrotizing pneomococcal pneumonia in childhood. Pediatr Pulmonol. 1999;28(3):217-21.

5. Kerem E, Bar Ziv Y, Rudenski B, et al. Bacteremic necrotizing pneumococcal pneumonia in children. Am J Respir Crit Care Med. 1994;149(1):242-4.

6. Velhote, CEP, Velhote, MCP, Velhote TFO. Decorticação pleural precoce no tratamento do empiema pleural complicado na criança. Rev Col Bras Cir. 2000; 27(1):41-4.

7. Hodina $\mathrm{M}$, Hanquinet $\mathrm{S}$, Cotting $\mathrm{J}$, et al. Imaging of cavitary necrosis in complicated childhood pneumonia. Eur Radiol. 2002;12(2):391-6. 
8. Patton RM, Abrams RS, Gauderer MW. Is thoracoscopically aided pleural debridement advantageous in children? Am Surg. 1999;65(1):69-72.

9. Cunniffe MG, Maguire D, McAnena OJ, et al. Video-assisted thoracoscopic surgery in the management of loculated empyema. Surg Endosc. 2000;14(2):175-8.

10. Liu HP, Hsieh MJ, Lu HI, et al. Thoracoscopic-assisted management of postpneumonic empyema in children refractory to medical response. Surg Endosc. 2002;16(11):1612-4.

11. IPEG guidelines for surgical treatment of empyema and related pleural diseases. Pediatr Endosurg Innov Techn. 2003;7(2):205-9.

12. Nizamettin K, Çelebi S, Gürpinar A, et al. Management of thoracic empyema in children. Pediatr Surg Int. 2002;18(1):21-3.

13. Cohen G, Hjortdal V, Ricci M, et al. Primary thoracoscopic treatment of empyema in children. J Thorac Cardiovasc Surg. 2003;125(1):79-83; discussion 83-4.
14. Striffeler H, Gugger M, Im Hof V, et al. Video-assisted thoracoscopic surgery for fibrinopurulent pleural empyema in 67 patients. Ann Thorac Surg. 1998;65(2):319-23.

15. Bouros D, Antoniou KM, Chalkiadakis G, et al. The role of video-assisted thoracoscopic surgery in the treatment of parapneumonic empyema after failure of fibrinolytics. Surg Endosc. 2002;16(1):151-4.

16. Gates RL, Caniano DA, Hayes JR, et al. Does VATS provide optimal treatment of empyema in children? A systematic review. J Pediatr Surg. 2004;39(3):381-6.

Endereço para correspondência:

Dr. Carlos Eduardo Prieto Velhote

Rua Carlos Weber 1389 Apto. 114

Alto da Lapa - São Paulo - CEP 05303-000

E-mail: cevelhot@terra.com.br 